\title{
A Bivariate Law of Iterated Logarithm for Partial Sums and Delayed Sums
}

\author{
R. Vasudeva* and Gooty Divanji*
}

Department of Studies in Statistics, Manasagangothri, University of Mysore, Mysore - 570006 - Karnataka - India

\begin{abstract}
When the random variables are positive strictly stable, we obtain a bivariate law of iterated logarithm for the vector of partial sums and delayed sums.
\end{abstract}

Key Words: Law of iterated logarithm, bivariate summands, stable laws.

\section{INTRODUCTION}

Let $\left\{X_{n}, n \geq 1\right\}$ be a sequence of independent identically distributed (i.i.d) positive strictly stable random variables (r.v.s) with exponent $\alpha, 0<\alpha<1$. Set $\mathrm{S}_{\mathrm{n}}=\sum_{\mathrm{k}=1}^{\mathrm{n}} \mathrm{X}_{\mathrm{k}}$ and $T_{n}=S_{n+a_{n}}-S_{n}$, where $\left\{a_{n}\right\}$ is non - decreasing sequence of positive integers. Write $\xi_{n}=\left\{\left(\frac{S_{n}}{n^{1 / \alpha}}\right)^{\theta_{n}},\left(\frac{T_{n}}{a_{n}{ }^{1 / \alpha}}\right)^{\gamma_{n}}\right\}$, where $\theta_{\mathrm{n}}=(\log \log n)^{-1}$ and $\gamma_{\mathrm{n}}=\left(\log \frac{\mathrm{n}}{\mathrm{a}_{\mathrm{n}}}+\log \log \mathrm{n}\right)^{-1}$.

When $\left(\mathrm{S}_{1, \mathrm{n}}\right)$ and $\left(\mathrm{S}_{2, \mathrm{n}}\right)$ are independent copies of $\left(\mathrm{S}_{\mathrm{n}}\right)$, the authors in [1] have obtained the set of all limit points of the sequence $\left\{\left(\frac{\mathrm{S}_{1, \mathrm{n}}}{\mathrm{n}^{1 / \alpha}}\right)^{\theta_{\mathrm{n}}},\left(\frac{\mathrm{S}_{2, \mathrm{n}}}{\mathrm{n}^{1 / \alpha}}\right)^{\theta_{\mathrm{n}}}\right\}$. In this paper, under different conditions on $\left(a_{n}\right)$,

we obtain the almost sure limit sets of the sequence $\left\{\xi_{\mathrm{n}}, \mathrm{n} \geq 1\right\}$. A careful observation tells that the limit sets change with the rate of growth of $a_{n}$ in comparison with n.

The LIL in this paper is based on the right tail of the d.f. or the probability of occurrences of large values (following power law) in spirit, is on the lines of [2]. In [3], LIL has been obtained for $\left\{\frac{\mathrm{S}_{\mathrm{n}}}{\alpha_{\mathrm{n}} \mathrm{n}^{1 / \alpha}}, \frac{\mathrm{T}_{\mathrm{n}}}{\beta_{\mathrm{n}} \mathrm{a}_{\mathrm{n}}{ }^{1 / \alpha}}\right\}$, for suitable choices

*Address correspondence to these authors at the Department of Studies in Statistics, Manasagangothri, University of Mysore, Mysore - 570006 Karnataka - India; Tel: 091-821-2419853; Fax: 0091 - 821 - 2419301; E-mails: vasudeva.rasbagh@gmail.com and gootydivan@yahoo.co.in Mathematical Subject Classification (MSC): Primary 60F15 of $\alpha_{n}$ and $\beta_{n}$, which depend on the behavior of the d.f. near the tail approaching zero (exponentially fast). As such, the normalization in [3] is linear and in the present paper, it is power normalization.

Through out this paper $[\mathrm{x}]$ stands for the largest integer which is less than or equal to a positive number $\mathrm{x}$, where as a.s and i.o mean almost surely and infinitely often respectively. C, $\varepsilon$ (small), $\mathrm{k}$ (integer) and $\mathrm{N}$ (integer), with or with out a suffix, stand for positive constants. For any sequence $\left(Y_{n}\right)$ of r.v.s, lim sup (inf) $Y_{n}=\alpha(\beta)$ is to be read as lim sup $Y_{n}=\alpha$ and $\lim \inf Y_{n}=\beta$.

In the next section we present some preliminary results. The almost sure limit sets of the vector sequence $\left\{\xi_{n}, n \geq 1\right\}$ are obtained in the last section. We assume that $\frac{a_{n}}{n} \sim b_{n}$, where $\left(b_{n}\right)$ is non-increasing. For instance, if $a_{n}=\left[n^{p}\right], 0<p$ $<1$ then $\frac{a_{n}}{n}=\frac{\left[n^{p}\right]}{n}$. Taking $b_{n}=\frac{n^{p}}{n}$, one can see that $\frac{a_{n}}{n} \sim b_{n}$ and $\left(b_{n}\right)$ is non-increasing. However, taking $p=1 / 2$, one can observe that $\frac{\mathrm{a}_{\mathrm{n}}}{\mathrm{n}}$ fails to be non-increasing. Similar justification holds when $a_{n}=[n p]$. Here $b_{n}=\frac{n p}{n}=p, 0<p<1$.

\section{LEMMAS}

\section{Lemma 1 (Extended Borel - Cantelli Lemma)}

Let $\left(E_{n}\right)$ be a sequence of events in a common probability space. if (i) $\sum_{n=1}^{\infty} \mathrm{P}\left(\mathrm{E}_{\mathrm{n}}\right)=\infty$ and

(ii) $\operatorname{Liminf}_{n \rightarrow \infty} \frac{\sum_{k=1}^{n} \sum_{s=1}^{n} P\left(E_{k} C E_{s}\right)}{\left(\sum_{k=1}^{n} P\left(E_{k}\right)\right)^{2}} \geq C$, then $P\left(E_{k}\right.$ i.o $) \geq C^{-1}$. 
For proof, see [4, lemma P3, p.317].

\section{Lemma 2}

Let $\left(A_{n}\right)$ be a sequence of events in a common probability space. If $\mathrm{P}\left(\mathrm{A}_{\mathrm{n}}\right) \rightarrow 0$ and $\sum_{n=1}^{\infty} \mathrm{P}\left(\mathrm{A}_{\mathrm{n}} \cap \mathrm{A}_{\mathrm{n}+1}^{\mathrm{c}}\right)<\infty$ then $\mathrm{P}\left(\mathrm{A}_{\mathrm{n}}\right.$ i.o $)$ $=0$. For proof see $\left[5\right.$, lemma $1^{*}$, p.385].

\section{Lemma 3}

$$
\lim \sup _{n \rightarrow \infty}(\inf )\left(\frac{S_{n}}{n^{1 / \alpha}}\right)^{\theta_{n}}=e^{1 / \alpha}(1) \text { a.s }
$$

For proof see [1]

\section{Lemma 4}

Let $\left\{X_{n}, n \geq 1\right\}$ be i.i.d positive strictly stable r. v. s with exponent $\alpha, 0<\alpha<1$. Let $\left(a_{n}\right), 0<a_{n} \leq n$, be a sequence of non - decreasing integers with $\frac{a_{n}}{n} \sim b_{n}$, where $b_{n}$ is non increasing. Then

$\left.\operatorname{Lim}_{n \rightarrow \infty} \operatorname{Inf}_{\left(\frac{T_{n}}{a_{n}^{1 / a}}\right.}\right)^{\tilde{a}_{n}}=1 \quad$ a.s

\section{Proof}

To prove the lemma it suffices to show that for any $\varepsilon>0$,

$$
P\left(\frac{T_{n}}{a_{n}^{1 / \alpha}} \leq\left(\frac{n}{a_{n}} \log n\right)^{\varepsilon} \text { i.o }\right)=1
$$

and

$$
\mathrm{P}\left(\frac{\mathrm{T}_{\mathrm{n}}}{\mathrm{a}_{\mathrm{n}}^{1 / \alpha}} \leq\left(\frac{\mathrm{n}}{\mathrm{a}_{\mathrm{n}}} \log \mathrm{n}\right)^{-\varepsilon} \text { i.o }\right)=0
$$

The fact that $X_{n}$ 's are positive valued strictly stable r.v.s implies that $\frac{T_{n}}{a_{n}^{1 / \alpha}}$ and $X_{1}$ are identically distributed. Observe that $\frac{\mathrm{n}}{\mathrm{a}_{\mathrm{n}}}$ is non-decreasing implies $\left(\frac{\mathrm{n}}{\mathrm{a}_{\mathrm{n}}} \log \mathrm{n}\right)^{\varepsilon} \rightarrow \infty$ as $\mathrm{n} \rightarrow$ $\begin{array}{ll}\infty & \text { We } \\ \left.\mathrm{T}_{\mathrm{n}} \leq \mathrm{a}_{\mathrm{n}}^{1 / \alpha}\left(\frac{\mathrm{n}}{\mathrm{a}_{\mathrm{n}}} \log \mathrm{n}\right)^{\varepsilon}\right)=\mathrm{P}\left(\mathrm{X} \leq\left(\frac{\mathrm{n}}{\mathrm{a}_{\mathrm{n}}} \log \mathrm{n}\right)^{\varepsilon}\right), & \text { hhich }\end{array}$ implies that

$$
\operatorname{Lim}_{n \rightarrow \infty} P\left(T_{n} \leq a_{n}^{1 / \alpha}\left(\frac{n}{a_{n}} \log n\right)^{\varepsilon}\right)=1
$$

Note that

$$
\begin{aligned}
\mathrm{P}\left(\mathrm{T}_{\mathrm{n}} \leq \mathrm{a}_{\mathrm{n}}^{1 / \alpha}\left(\frac{\mathrm{n}}{\mathrm{a}_{\mathrm{n}}} \log \mathrm{n}\right)^{\varepsilon} \text { i.o }\right) & =\mathrm{P}\left(\bigcap_{\mathrm{n}=1 \mathrm{~m}=\mathrm{n}}^{\infty} \bigcup_{\mathrm{m}}^{\infty}\left(\mathrm{T}_{\mathrm{m}} \leq \mathrm{a}^{1 / \alpha}\left(\frac{\mathrm{m}}{\mathrm{a}_{\mathrm{m}}} \log \mathrm{m}\right)^{\varepsilon}\right)\right) \\
= & \operatorname{Lim}_{\mathrm{n} \rightarrow \infty} \mathrm{P}\left(\bigcup_{\mathrm{m}=\mathrm{n}}^{\infty} \mathrm{T}_{\mathrm{m}} \leq \mathrm{a}_{\mathrm{m}}^{1 / \alpha}\left(\frac{\mathrm{m}}{\mathrm{a}_{\mathrm{m}}} \log \mathrm{m}\right)^{\varepsilon}\right) \\
& \geq \operatorname{Lim}_{\mathrm{n} \rightarrow \infty} \mathrm{P}\left(\mathrm{T}_{\mathrm{n}} \leq \mathrm{a}_{\mathrm{n}}^{1 / \alpha}\left(\frac{\mathrm{n}}{\mathrm{a}_{\mathrm{n}}} \log \mathrm{n}\right)^{\varepsilon}\right)
\end{aligned}
$$

From (3), we get $\mathrm{P}\left(\mathrm{T}_{\mathrm{n}} \leq \mathrm{a}_{\mathrm{n}}^{1 / \alpha}\left(\frac{\mathrm{n}}{\mathrm{a}_{\mathrm{n}}} \log \mathrm{n}\right)^{\varepsilon}\right.$ i.o $)=1$ and hence the proof of (1) is complete.

Now we will complete the proof of the Lemma by showing that for any $\varepsilon \in(0,1)$,

$\mathrm{P}\left(\mathrm{T}_{\mathrm{n}} \leq \mathrm{a}_{\mathrm{n}}^{1 / \alpha}\left(\frac{\mathrm{n}}{\mathrm{a}_{\mathrm{n}}} \log \mathrm{n}\right)^{-\varepsilon}\right.$ i.o $)=0$. We define $\mathrm{n}_{\mathrm{k}+1}$ as the smallest integer greater than or equal to $n_{k}+\frac{a_{n_{k}}}{\log \log a_{n_{k}}}, k$ $=1,2, \ldots$; and $\mathrm{n}_{1}$ as first integer $\mathrm{n}$ such that $\mathrm{a}_{\mathrm{n}}>3$. Let $\mathrm{C}_{1, \mathrm{n}}$, $\mathrm{D}_{1, \mathrm{k}}$ and $\mathrm{E}_{1, \mathrm{k}}$ denote the

events

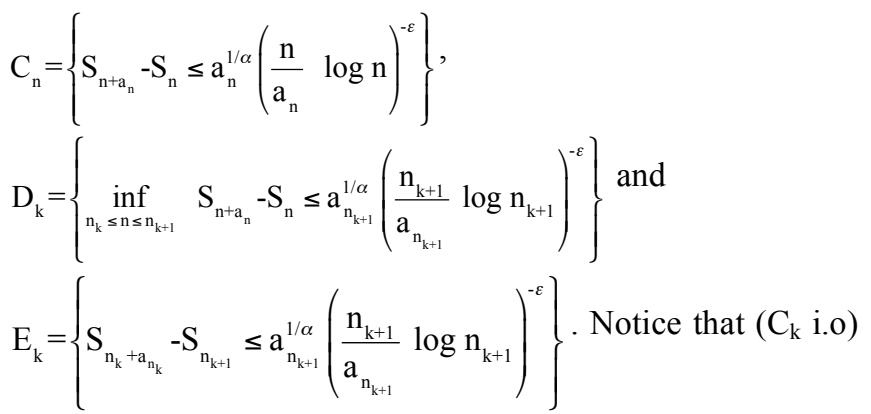

$\left(C_{k}\right.$ i.o $) \subset\left(D_{k}\right.$ i.o $) \subset\left(E_{k}\right.$ i.o $)$. Hence in order to prove (2), it is enough if we show that $\quad \mathrm{P}\left(\mathrm{E}_{\mathrm{k}}\right.$ i.o $)=0$

We have,

$P\left(E_{k}\right)=P\left(S_{n_{k}+a_{n_{k}}}-S_{n_{k+1}} \leq a_{n_{k+1}}^{1 / \alpha}\left(\frac{n_{k}}{a_{n_{k}}} \log n_{k}\right)^{-\varepsilon}\right)=$

$\mathrm{P}\left(\mathrm{X}_{1} \leq \frac{\mathrm{a}_{\mathrm{n}_{\mathrm{k}+1}^{1 / \varepsilon}}}{\left(\mathrm{n}_{\mathrm{k}}+\mathrm{a}_{\mathrm{n}_{\mathrm{k}}}-\mathrm{n}_{\mathrm{k}+1}\right)^{1 / \alpha}}\left(\frac{\mathrm{n}_{\mathrm{k}}}{\mathrm{a}_{\mathrm{n}_{\mathrm{k}}}} \log \mathrm{n}_{\mathrm{k}}\right)^{-\varepsilon}\right)$

We observe that for $\mathrm{k} \geq \mathrm{k}_{0}$

$\frac{a_{n_{k+1}}^{1 / \alpha}}{\left(n_{k}+a_{n_{k}}-n_{k+1}\right)^{1 / \alpha}}\left(\frac{n_{k}}{a_{n_{k}}} \log n_{k}\right)^{-\varepsilon} \leq 2\left(\frac{a_{n_{k+1}}}{a_{n_{k}}}\right)^{1 / \alpha}\left(\frac{n_{k+1}}{n_{k}} \log n_{k+1}\right)^{-\varepsilon}$.

The fact that $a_{n} / n$ is non - increasing as $n \rightarrow \infty$ implies that $\frac{a_{n_{k+1}}}{n_{k+1}} \leq \frac{a_{n_{k}}}{n_{k}}$ or $\frac{a_{n_{k+1}}}{a_{n_{k}}} \leq \frac{n_{k+1}}{n_{k}}$. Again from the relation 
$\mathrm{n}_{\mathrm{k}+1}=\mathrm{n}_{\mathrm{k}}+\frac{\mathrm{a}_{\mathrm{n}_{\mathrm{k}}}}{\log \log \mathrm{a}_{\mathrm{n}_{\mathrm{k}}}}$, one can show that $\frac{\mathrm{n}_{\mathrm{k}+1}}{\mathrm{n}_{\mathrm{k}}} \rightarrow 1$ as $\mathrm{k} \rightarrow \infty$. Hence for a given $\varepsilon_{1}>0$ there exists $k_{1}$ such that $\frac{\mathrm{a}_{\mathrm{n}_{\mathrm{k}+1}}}{\mathrm{a}_{\mathrm{n}_{\mathrm{k}}}} \leq\left(1+\varepsilon_{1}\right)$, for all $\mathrm{k} \geq \mathrm{k}_{1}$.

Consequently for all $\mathrm{k} \geq \mathrm{k}_{1}$,

$\mathrm{P}\left(\mathrm{E}_{\mathrm{k}}\right) \leq \mathrm{P}\left(\mathrm{X}_{1} \leq\left(1+\varepsilon_{1}\right)\left(\frac{\mathrm{n}_{\mathrm{k}}}{\mathrm{a}_{\mathrm{n}_{\mathrm{k}}}} \log \mathrm{n}_{\mathrm{k}}\right)^{-\varepsilon}\right)$. From theorem 1 of $[6$, p.424], one can now get

$\mathrm{P}\left(\mathrm{E}_{\mathrm{k}}\right) \leq \mathrm{C} \exp \left\{-\left(\left(1+\varepsilon_{1}\right)\left(\frac{\mathrm{n}_{\mathrm{k}}}{\mathrm{a}_{\mathrm{n}_{\mathrm{k}}}} \log \mathrm{n}_{\mathrm{k}}\right)^{-\varepsilon}\right)^{-\alpha}\right\}$, for some $\mathrm{C}>0$.

Let $\left(1+\varepsilon_{1}\right)^{-\alpha}=\left(1-\varepsilon_{2}\right), \varepsilon_{2}>0$. Then

$\mathrm{P}\left(\mathrm{E}_{\mathrm{k}}\right) \leq \mathrm{C} \exp \left\{-\left(1-\varepsilon_{2}\right)\left(\frac{\mathrm{n}_{\mathrm{k}}}{\mathrm{a}_{\mathrm{n}_{\mathrm{k}}}} \log \mathrm{n}_{\mathrm{k}}\right)^{\alpha \varepsilon}\right\}$.

We now claim that, for some $\varepsilon_{3}>0$, $\exp \left\{-\left(1-\varepsilon_{2}\right)\left(\frac{\mathrm{n}_{\mathrm{k}}}{\mathrm{a}_{\mathrm{n}_{\mathrm{k}}}} \log \mathrm{n}_{\mathrm{k}}\right)^{\alpha \varepsilon}\right\}=\mathrm{o}\left(\frac{\mathrm{a}_{\mathrm{n}_{\mathrm{k}}}}{\mathrm{n}_{\mathrm{k}}} \frac{1}{\left(\log \mathrm{n}_{\mathrm{k}}\right)^{\left(1+\varepsilon_{3}\right)}}\right)$.

$$
\frac{\exp \left\{-\left(1-\varepsilon_{2}\right)\left(\frac{n_{k}}{a_{n_{k}}} \log n_{k}\right)^{\alpha \varepsilon}\right\}}{\frac{a_{n_{k}}}{n_{k}} \frac{1}{\left(\log n_{k}\right)^{\left(1+\varepsilon_{3}\right)}}}=\frac{\frac{n_{k}}{a_{n_{k}}}\left(\log n_{k}\right)^{\left(1+\varepsilon_{3}\right)}}{\exp \left\{\left(1-\varepsilon_{2}\right)\left(\frac{n_{k}}{a_{n_{k}}} \log n_{k}\right)^{\alpha \varepsilon}\right\}} .
$$

have

The fact that

$\frac{n_{k}}{a_{n_{k}}}\left(\log n_{k}\right)^{\left(1+\varepsilon_{3}\right)} \rightarrow \infty \quad$ immediately implies that $\frac{\frac{n_{k}}{a_{n_{k}}}\left(\log n_{k}\right)^{\left(1+\varepsilon_{3}\right)}}{\exp \left\{\left(1-\varepsilon_{2}\right)\left(\frac{n_{k}}{a_{n_{k}}} \log n_{k}\right)^{\alpha \varepsilon}\right\}} \rightarrow 0$ as $n \rightarrow \infty$ and the claim is justified. Hence there exists some $\mathrm{C}_{1}(>\mathrm{C})$ and $\mathrm{k}_{2}$ such that for all $\mathrm{k} \geq \mathrm{k}_{2}$,

$P\left(E_{k}\right) \leq C_{1}\left(\frac{a_{n_{k}}}{n_{k}}\right)\left(\frac{1}{\left(\log n_{k+1}\right)^{\left(1+\varepsilon_{3}\right)}}\right)=C_{1} \frac{a_{n_{k}}}{n_{k}} \frac{1}{\left(\log n_{k}\right)^{\left(1+\varepsilon_{3}\right)}}$. Now $\mathrm{n}_{\mathrm{k}+1}=\mathrm{n}_{\mathrm{k}}+\frac{\mathrm{a}_{\mathrm{n}_{\mathrm{k}}}}{\log \log \mathrm{a}_{\mathrm{n}_{\mathrm{k}}}}$ gives $\mathrm{a}_{\mathrm{n}_{\mathrm{k}}}=\left(\mathrm{n}_{\mathrm{k}+1}-\mathrm{n}_{\mathrm{k}}\right) \log \log \mathrm{a}_{\mathrm{n}_{\mathrm{k}}}$.

Using the fact that $\frac{\log \log a_{n_{k}}}{\left(\log n_{k}\right)^{\frac{\varepsilon_{3}}{2}}} \rightarrow 0$, as $n \rightarrow \infty$. We can find $\left(\log \mathrm{n}_{\mathrm{k}}\right)^{\frac{\varepsilon_{3}}{2}}$

$a k_{3} \geq k_{2}$ such that for all $k \geq k_{3}$,
$P\left(E_{k}\right) \leq C_{1}\left(\frac{n_{k+1}-n_{k}}{n_{k}}\right)\left(\frac{\log \log a_{n_{k}}}{\left.\left(\log n_{k}\right)^{\left(1+\varepsilon_{3}\right.}\right)}\right) \leq C_{1}\left(\frac{n_{k+1}-n_{k}}{n_{k}}\right)\left(\frac{1}{\left.\left(\log n_{k}\right)^{\left(1+\frac{\varepsilon_{3}}{2}\right.}\right)}\right)$.

From the relation $\frac{\mathrm{n}_{\mathrm{k}+1}}{\mathrm{n}_{\mathrm{k}}} \rightarrow 1$

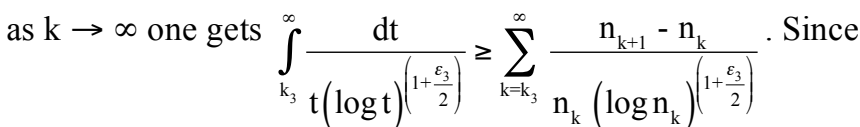

$\int_{\mathrm{k}_{3}}^{\infty} \frac{\mathrm{dt}}{\mathrm{t}(\log \mathrm{t})^{\left(1+\frac{\varepsilon_{3}}{2}\right)}}<\infty, \quad$ one gets $\quad \sum_{\mathrm{k}=\mathrm{k}_{3}}^{\infty} \frac{\mathrm{n}_{\mathrm{k}+1}-\mathrm{n}_{\mathrm{k}}}{\mathrm{n}_{\mathrm{k}}\left(\log \mathrm{n}_{\mathrm{k}}\right)^{\left(1+\frac{\varepsilon_{3}}{2}\right)}}<\infty$ or $\sum_{\mathrm{k}=\mathrm{k}_{3}}^{\infty} \mathrm{P}\left(\mathrm{E}_{1, \mathrm{k}}\right)<\infty$, which in turn establishes (4) by appealing to B.C lemma. Hence the proof of the Lemma is complete.

\section{Lemma 5}

$\operatorname{Lim}_{n \rightarrow \infty} \operatorname{Sup}_{n}\left(\frac{T_{n}}{a_{n}^{1 / \alpha}}\right)^{\gamma_{n}}=e^{1 / \alpha} \quad$ a.s

Proof

The proof is on lines of [7] and hence is omitted.

3. LIMIT POINTS OF THE SEQUENCE $\left\{\xi_{\mathrm{n}}, \mathrm{n} \geq 1\right\}$

We observe by lemmas 3, 4 and 5 that the set of a.s. limit points of the sequences $\left(\xi_{\mathrm{n}}\right)$ is included in $\left[1, \mathrm{e}^{1 / \alpha}\right] \times\left[1, \mathrm{e}^{1 / \alpha}\right]$. We will devote this section for the identification of the limit sets of the sequence $\left\{\xi_{n}, n \geq 1\right\}$, when $a_{n}=\left[n^{p}\right], 0<p<1, a_{n}=$ [np], $0<\mathrm{p}<1$ and $\mathrm{a}_{\mathrm{n}}=\left[\frac{\mathrm{n}}{(\log n)^{\mathrm{q}}}\right], \mathrm{q}>0$. Define $\mathrm{A}_{1}=\left\{\left[1, \mathrm{e}^{\frac{1}{\alpha}}\right] \mathrm{X}\left[1, \mathrm{e}^{\frac{1}{\alpha}}\right]\right\}$ and $\mathrm{A}_{2}=\left\{\left(\mathrm{e}^{\frac{\mathrm{u}}{\alpha}}, \mathrm{e}^{\frac{\mathrm{v}}{\alpha}}\right): 0<\mathrm{u}, \mathrm{v}<1, \mathrm{u}+\mathrm{v} \leq 1\right\}$.

We will show that a.s. limit set of $\left\{\xi_{n}, n \geq 1\right\}$ coincide with $A_{1}$, and $A_{2}$ respectively, when

$\mathrm{a}_{\mathrm{n}}=\left[\mathrm{n}^{\mathrm{p}}\right], 0<\mathrm{p}<1, \mathrm{a}_{\mathrm{n}}=[\mathrm{np}], 0<\mathrm{p}<1$. When $a_{n}=\left[\frac{n}{(\log n)^{q}}\right], q>0$, we show that the a.s. limit set is

again $A_{2}$. Hence the limit sets change with the rate of growth of $a_{n}$ in comparison with $n$.

\section{Theorem 1}

When $\mathrm{a}_{\mathrm{n}}=\left[\mathrm{n}^{\mathrm{p}}\right], 0<\mathrm{p}<1$, the set of all a.s. limit points of the sequence $\quad\left\{\xi_{n}, n \geq 1\right\}$ coincides with $\mathrm{A}_{1}=\left\{\left[1, \mathrm{e}^{\frac{1}{\alpha}}\right] \mathrm{X}\left[1, \mathrm{e}^{\frac{1}{\alpha}}\right]\right\}$.

Proof

The fact that the limit set of the sequence $\left\{\xi_{n}, n \geq 1\right\}$ is contained in $A_{1}$ is immediate from the lemmas 3, 4 and 5. 
Hence the proof will be complete once we establish that every element of $A_{1}$ is a limit point of $\left\{\xi_{n}, n \geq 1\right\}$. In other words for $\left(\mathrm{e}^{\frac{\mathrm{u}}{\alpha}}, \mathrm{e}^{\frac{v}{\alpha}}\right) \in \mathrm{A}_{1}$ with $0<\mathrm{u}, \mathrm{v}<1$ and $0<\varepsilon<\min (\mathrm{u}$, v), we have to show that,

$$
\mathrm{P}\left(\xi_{\mathrm{n}} \in\left(\mathrm{e}^{\frac{\mathrm{u}-\varepsilon}{\alpha}}, \mathrm{e}^{\frac{\mathrm{u}+\varepsilon}{\alpha}}\right) X\left(\mathrm{e}^{\frac{\mathrm{v}-\varepsilon}{\alpha}}, \mathrm{e}^{\frac{\mathrm{v}+\varepsilon}{\alpha}}\right) \mathrm{i} . \mathrm{o}\right)=1
$$

Note that $\mathrm{a}_{\mathrm{n}}=\left[\mathrm{n}^{\mathrm{p}}\right], 0<\mathrm{p}<1$ implies that $\gamma_{\mathrm{n}} \approx(1-\mathrm{p}) \log \mathrm{n}$. We prove that for some $\mathrm{d}>0$, $\mathrm{P}\left(\xi_{\mathrm{n}} \in\left(\mathrm{e}^{\frac{\mathrm{u}-\varepsilon}{\alpha}}, \mathrm{e}^{\frac{\mathrm{u}+\varepsilon}{\alpha}}\right) \mathrm{X}\left(\mathrm{e}^{\frac{\mathrm{v}-\varepsilon}{\alpha}}, \mathrm{e}^{\frac{\mathrm{v}+\varepsilon}{\alpha}}\right)\right.$ i.o $) \geq \mathrm{d}>0$. This is done by applying lemma1. By Hewitt - Savage zero - one law (5) will be established.

Define $\mathrm{n}_{\mathrm{k}}=\left[\mathrm{k}^{\frac{1}{(1-\mathrm{p}) \mathrm{v}}}\right]$

and

$$
\begin{aligned}
& \mathrm{H}_{\mathrm{k}}=\left\{\mathrm{n}_{\mathrm{k}}^{1 / \alpha}\left(\log \mathrm{n}_{\mathrm{k}}\right)^{\frac{\mathrm{u}-\varepsilon}{\alpha}} \leq \mathrm{S}_{\mathrm{n}_{\mathrm{k}}} \leq \mathrm{n}_{\mathrm{k}}^{1 / \alpha}\left(\log \mathrm{n}_{\mathrm{k}}\right)^{\frac{\mathrm{u}+\varepsilon}{\alpha}},\right. \\
& \left.\mathrm{n}_{\mathrm{k}}^{\frac{\mathrm{p}}{\mathrm{a}}+\frac{\mathrm{v}(1-\mathrm{p})}{\mathrm{a}}} \leq \mathrm{T}_{\mathrm{n}_{\mathrm{k}}} \leq \mathrm{n}_{\mathrm{k}}^{\frac{\mathrm{p}}{\mathfrak{a}}+\frac{(\mathrm{v}+\mathrm{a})(1-\mathrm{p})}{\mathfrak{a}}}\right\}
\end{aligned}
$$

Since $X_{i}$ 's are positive valued strictly stable r.v.s and $\left(S_{n}\right)$ and $\left(T_{n}\right)$ are independent, we have

$$
\begin{aligned}
& \mathrm{P}\left(\mathrm{H}_{\mathrm{k}}\right)=\mathrm{P}\left(\left(\log \mathrm{n}_{\mathrm{k}}\right)^{\frac{\mathrm{u}-\varepsilon}{\alpha}} \leq \mathrm{X}_{1} \leq\left(\log \mathrm{n}_{\mathrm{k}}\right)^{\frac{\mathrm{u}+\varepsilon}{\alpha}}\right) \mathrm{P} . \\
& \left(\mathrm{n}_{\mathrm{k}}^{\frac{\mathrm{v}(1-\mathrm{p})}{\alpha}} \leq \mathrm{X}_{2} \leq \mathrm{n}_{\mathrm{k}}^{\frac{(\mathrm{v}+\varepsilon)(1-\mathrm{p})}{\alpha}}\right)
\end{aligned}
$$

Since $\mathrm{X}_{\mathrm{i}}$ 's are positive strictly stable r.v.s, we have $\mathrm{P}(\mathrm{X}$ $\geq \mathrm{x}) \sim \mathrm{O}\left(\mathrm{x}^{-\alpha}\right)$

Using (7), we note that there exists constant $C_{1}(>0)$ and $\mathrm{k}_{1}$ such that for all $\mathrm{k} \geq \mathrm{k}_{1}$,

$\mathrm{P}\left(\mathrm{H}_{\mathrm{k}}\right) \geq \frac{\mathrm{C}_{1}}{\mathrm{k}(\log \mathrm{k})^{(\mathrm{u}-\varepsilon)}}$

which implies that $\sum_{\mathrm{k}=\mathrm{k}_{1}}^{\infty} \mathrm{P}\left(\mathrm{H}_{\mathrm{k}}\right)=\infty$. Observe that

$$
\frac{\sum_{\mathrm{k}=1}^{\mathrm{n}} \sum_{\mathrm{s}=1}^{\mathrm{n}} \mathrm{P}\left(\mathrm{H}_{\mathrm{k}} \cap \mathrm{H}_{\mathrm{s}}\right)}{\left(\sum_{\mathrm{k}=1}^{\mathrm{n}} \mathrm{P}\left(\mathrm{H}_{\mathrm{k}}\right)\right)^{2}}=\frac{2 \sum_{\mathrm{k}=1}^{\mathrm{n}} \sum_{\mathrm{s}=\mathrm{k}+1}^{\mathrm{n}-1} \mathrm{P}\left(\mathrm{H}_{\mathrm{k}} \cap \mathrm{H}_{\mathrm{s}}\right)}{\left(\sum_{\mathrm{k}=1}^{\mathrm{n}} \mathrm{P}\left(\mathrm{H}_{\mathrm{k}}\right)\right)^{2}}+\frac{1}{\sum_{\mathrm{k}=1}^{\mathrm{n}} \mathrm{P}\left(\mathrm{H}_{\mathrm{k}}\right)}
$$

In order to establish (9) of E.B.C lemma, we proceed as under. For $\mathrm{s}>\mathrm{k}$, let

$$
\begin{aligned}
& \mathrm{L}_{1}=\left\{\mathrm{n}_{\mathrm{k}}^{1 / \alpha}\left(\log \mathrm{n}_{\mathrm{k}}\right)^{\frac{\mathrm{u}-\varepsilon}{\alpha}} \leq \mathrm{S}_{\mathrm{n}_{\mathrm{k}}} \leq \mathrm{n}_{\mathrm{k}}^{1 / \alpha}\left(\log \mathrm{n}_{\mathrm{k}}\right)^{\frac{\mathrm{u}+\varepsilon}{\alpha}}\right\} \text {, } \\
& \mathrm{L}_{2}=\left\{\mathrm{n}_{\mathrm{k}}^{\frac{\mathrm{p}}{\alpha}+\frac{\mathrm{v}(1-\mathrm{p})}{\alpha}} \leq \mathrm{T}_{\mathrm{n}_{\mathrm{k}}} \leq \mathrm{n}_{\mathrm{k}}^{\frac{\mathrm{p}}{\alpha}+\frac{(\mathrm{v}+\varepsilon)(1-\mathrm{p})}{\alpha}}\right\} \\
& \mathrm{L}_{3}=\left\{\mathrm{n}_{\mathrm{s}}^{1 / \alpha}\left(\log \mathrm{n}_{\mathrm{s}}\right)^{\frac{\mathrm{u}-\varepsilon}{\alpha}} \leq \mathrm{S}_{\mathrm{n}_{\mathrm{s}}} \leq \mathrm{n}_{\mathrm{s}}^{1 / \alpha}\left(\log \mathrm{n}_{\mathrm{s}}\right)^{\frac{\mathrm{u}+\varepsilon}{\alpha}}\right\} \text {, } \\
& \mathrm{L}_{4}=\left\{\begin{array}{c}
\mathrm{n}_{\mathrm{s}}^{1 / \alpha}\left(\log \mathrm{n}_{\mathrm{s}}\right)^{\frac{\mathrm{u}-\varepsilon}{\alpha}}-\left(\mathrm{n}_{\mathrm{k}}^{1 / \alpha}\left(\log \mathrm{n}_{\mathrm{k}}\right)^{\frac{\mathrm{u}+\varepsilon}{\alpha}}+\mathrm{n}_{\mathrm{k}}^{\frac{\mathrm{p}}{\alpha}+\frac{(\mathrm{v}+\varepsilon)}{\alpha}(1-\mathrm{p})}\right) \leq \mathrm{S}_{\mathrm{n}_{\mathrm{s}}}-\left(\mathrm{S}_{\mathrm{n}_{\mathrm{k}}}+\mathrm{T}_{\mathrm{n}_{\mathrm{k}}}\right) \leq \\
\mathrm{n}_{\mathrm{s}}^{1 / \alpha}\left(\log \mathrm{n}_{\mathrm{s}}\right)^{\frac{\mathrm{u}-\varepsilon}{\alpha}}-\left(\mathrm{n}_{\mathrm{k}}^{1 / \alpha}\left(\log \mathrm{n}_{\mathrm{k}}\right)^{\frac{\mathrm{u}+\varepsilon}{\alpha}}+\mathrm{n}_{\mathrm{k}}^{\frac{\mathrm{p}}{\alpha}+\frac{\mathrm{v}}{\varepsilon}(1-\mathrm{p})}\right)
\end{array}\right\} \\
& \mathrm{L}_{5}=\left\{\mathrm{n}_{\mathrm{s}}^{\frac{\mathrm{p}}{\alpha}+\frac{\mathrm{v}(1-\mathrm{p})}{\alpha}} \leq \mathrm{T}_{\mathrm{n}_{\mathrm{s}}} \leq \mathrm{n}_{\mathrm{s}}^{\frac{\mathrm{p}}{\alpha}+\frac{(\mathrm{v}+\varepsilon)(1-\mathrm{p})}{\alpha}}\right\} \text {. }
\end{aligned}
$$

We have $\mathrm{E}_{\mathrm{k}} \cap \mathrm{E}_{\mathrm{s}}=\left\{\mathrm{L}_{1} \cap \mathrm{L}_{2} \cap \mathrm{L}_{3} \cap \mathrm{L}_{5}\right\} \subset\left\{\mathrm{L}_{1} \cap \mathrm{L}_{2} \cap\right.$ $\left.\mathrm{L}_{4} \cap \mathrm{L}_{5}\right\}$

$$
\text { Since } P\left(L_{3}\right)=P\left(\left(\log n_{s}\right)^{\frac{u}{\alpha}-\varepsilon} \leq X_{1} \leq\left(\log n_{s}\right)^{\frac{u}{\alpha}+\varepsilon}\right) \text {. }
$$

one can find constants $\mathrm{C}_{2}, \mathrm{k}_{2}$ such that for all $\mathrm{k} \geq \mathrm{k}_{2}$, $\mathrm{P}\left(\mathrm{L}_{3}\right) \geq \frac{\mathrm{C}_{2}}{\left(\log \mathrm{n}_{\mathrm{s}}\right)^{\mathrm{u}-\varepsilon}}$

Let $\mathrm{s}>\mathrm{k}(\log \mathrm{k})^{\lambda}, \lambda$ is sufficiently small compared to (1u), we have

$$
\begin{aligned}
& \mathrm{P}\left(\mathrm{L}_{4}\right) \leq \mathrm{P}\left(\mathrm{S}_{\mathrm{n}_{\mathrm{s}}}-\left(\mathrm{S}_{\mathrm{n}_{\mathrm{k}}}+\mathrm{T}_{\mathrm{n}_{\mathrm{k}}}\right) \geq \mathrm{n}_{\mathrm{s}}^{1 / \alpha}\left(\log \mathrm{n}_{\mathrm{s}}\right)^{\frac{\mathrm{u}-\mathrm{a}}{\hat{a}}}\right) \\
& =\mathrm{P}\left(\frac{\mathrm{S}_{\mathrm{n}_{\mathrm{s}}}-\left(\mathrm{S}_{\mathrm{n}_{\mathrm{k}}}+\mathrm{T}_{\mathrm{n}_{\mathrm{k}}}\right)}{\left(\mathrm{n}_{\mathrm{s}}-\left(\mathrm{n}_{\mathrm{k}}+\mathrm{n}_{\mathrm{k}}^{\mathrm{p}}\right)\right)^{1 / \alpha}} \geq \frac{\mathrm{n}_{\mathrm{s}}^{1 / \alpha}\left(\log \mathrm{n}_{\mathrm{s}}\right)^{\frac{\mathrm{u}-\varepsilon}{\alpha}}}{\left(\mathrm{n}_{\mathrm{s}}-\left(\mathrm{n}_{\mathrm{k}}+\mathrm{n}_{\mathrm{k}}^{\mathrm{p}}\right)\right)^{1 / \alpha}}\right) \leq \mathrm{P}\left(\mathrm{X}_{1} \geq \frac{\mathrm{n}_{\mathrm{s}}^{1 / \alpha}\left(\log \mathrm{n}_{\mathrm{s}}\right)^{\frac{\mathrm{u}-\varepsilon}{\alpha}}}{\left(1-\left(\mathrm{n}_{\mathrm{k}}+\mathrm{n}_{\mathrm{k}}^{\mathrm{p}}\right) \mathrm{n}_{\mathrm{s}}^{-1}\right)^{1 / \alpha}}\right) .
\end{aligned}
$$

Using the fact that $\mathrm{s}>\mathrm{k}(\log \mathrm{k})^{\lambda}$ one can note that

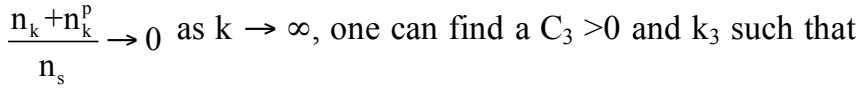
that for all $\mathrm{k} \geq \mathrm{k}_{3}, \mathrm{P}\left(\mathrm{L}_{4}\right) \leq \frac{\mathrm{C}_{3}}{\left(\log \mathrm{n}_{\mathrm{s}}\right)^{(\mathrm{u}-\varepsilon)}}$

From (11) and (12) one can notice that there exists a constant $\mathrm{C}_{4}>0$ such that for all

$\mathrm{k} \geq \mathrm{k}_{4}=\max \left(\mathrm{k}_{2}, \mathrm{k}_{3}\right), \quad \mathrm{P}\left(\mathrm{L}_{4}\right) \leq \mathrm{C}_{4} \mathrm{P}\left(\mathrm{L}_{3}\right)$.

From (9) we have for $s>k(\log k)^{\lambda}$ and for $k \geq k_{4}$,

$\mathrm{P}\left(\mathrm{H}_{\mathrm{k}} \cap \mathrm{H}_{\mathrm{s}}\right) \leq \mathrm{P}\left(\mathrm{L}_{1} \cap \mathrm{L}_{2} \cap \mathrm{L}_{4} \cap \mathrm{L}_{5}\right)=\mathrm{P}\left(\mathrm{L}_{1} \cap \mathrm{L}_{2}\right) \mathrm{P}\left(\mathrm{L}_{4}\right) \mathrm{P}\left(\mathrm{L}_{5}\right)$ $\leq \mathrm{C}_{4} \mathrm{P}\left(\mathrm{H}_{\mathrm{k}}\right) \mathrm{P}\left(\mathrm{L}_{3}\right) \mathrm{P}\left(\mathrm{L}_{5}\right)$

$\therefore \mathrm{P}\left(\mathrm{H}_{\mathrm{k}} \cap \mathrm{H}_{\mathrm{s}}\right) \leq \mathrm{C}_{4} \mathrm{P}\left(\mathrm{H}_{\mathrm{k}}\right) \mathrm{P}\left(\mathrm{H}_{\mathrm{s}}\right)$

Now for $(\mathrm{k}+1) \leq \mathrm{s} \leq \mathrm{k}(\log \mathrm{k})^{\lambda}$, using the inequality $\mathrm{P}\left(\mathrm{H}_{\mathrm{k}} \cap \mathrm{H}_{\mathrm{s}}\right) \geq \mathrm{P}\left(\mathrm{H}_{\mathrm{k}} \cap \mathrm{L}_{5}\right)$ and observing that $\left(\mathrm{S}_{\mathrm{n}_{\mathrm{k}}}\right),\left(\mathrm{T}_{\mathrm{n}_{\mathrm{k}}}\right)$ and $\left(\mathrm{T}_{\mathrm{n}_{\mathrm{s}}}\right)$ are independent, one gets $\mathrm{P}\left(\mathrm{H}_{\mathrm{k}} \cap \mathrm{H}_{\mathrm{s}}\right) \leq \mathrm{P}\left(\mathrm{H}_{\mathrm{k}}\right) \mathrm{P}\left(\mathrm{L}_{5}\right)$. 
Again using (7) and the fact $\mathrm{s} \geq \mathrm{k}+1$ one can find a constant $\mathrm{C}_{5}>0$ and $\mathrm{k}_{5}$ such that for all $\mathrm{k} \geq \mathrm{k}_{5}, \mathrm{P}\left(\mathrm{L}_{5}\right) \leq \frac{\mathrm{C}_{5}}{\mathrm{k}}$.

Hence for all $\mathrm{k} \geq \mathrm{k}_{5}, \mathrm{P}\left(\mathrm{H}_{\mathrm{k}} \cap \mathrm{H}_{\mathrm{s}}\right) \leq \frac{\mathrm{C}_{5}}{\mathrm{k}} \mathrm{P}\left(\mathrm{H}_{\mathrm{k}}\right)$

From (8) note that

$\mathrm{P}\left(\mathrm{H}_{\mathrm{k}}\right) \leq \mathrm{P}\left(\mathrm{X}_{1} \geq\left(\log \mathrm{n}_{\mathrm{k}}\right)^{\frac{\mathrm{u}-\varepsilon}{\alpha}}\right) \mathrm{P}\left(\mathrm{X}_{2} \geq \mathrm{n}_{\mathrm{k}}^{\frac{\mathrm{v}(1-\mathrm{p})}{\alpha}}\right)$. By applying (7) in

(15) one can find constants $\mathrm{C}_{6}>0$ and $\mathrm{k}_{6}$ such that for all $\mathrm{k} \geq$ $\mathrm{k}_{6}, \mathrm{P}\left(\mathrm{H}_{\mathrm{k}} \cap \mathrm{H}_{\mathrm{s}}\right) \leq \frac{\mathrm{C}_{6}}{\mathrm{k}^{2}(\log \mathrm{k})^{(\mathrm{u}-\mathrm{a})}}$.

Now $\sum_{k=k_{6}}^{n-1} \sum_{s=k+1}^{k(\log k) \lambda} P\left(H_{k} \cap H_{s}\right) \leq C_{6} \sum_{k=k_{6}}^{n-1} \frac{k(\log k)^{\lambda}}{k^{2}(\log k)^{(1-r)}}<C_{6} \sum_{k=k_{6}}^{n-1} \frac{1}{k(\log k)^{(1-\varepsilon-\lambda-\lambda)}} \cdot$

For $\mathrm{n} \quad \geq \quad \mathrm{N}_{1}, \quad$ we have $\sum_{k=k_{6}}^{n-1} \sum_{s=k+1}^{k(\log k)^{\lambda}} P\left(H_{k} \cap H_{s}\right) \leq C_{6}(\log n)^{1-(-(-1-\varepsilon-\lambda)}$. From (8) we have, for $\mathrm{n} \geq \mathrm{N}_{2},(16)$

$\sum_{k=k_{6}}^{n-1} P\left(H_{k}\right) \geq \sum_{k=k_{6}}^{n} \frac{C_{1}}{k(\log k)^{(u-\varepsilon)}} \geq C_{7}(\log n)^{1-(u-\varepsilon)}$, for some $C_{7}$ $>0$.

From (14), (16) and (17) one can get $\mathrm{C}_{8}>0$, $\operatorname{Liminf}_{n \rightarrow \infty} \frac{\sum_{k=1}^{n} \sum_{s=1}^{n} P\left(H_{k} \cap H_{s}\right)}{\left(\sum_{k=1}^{n} P\left(H_{k}\right)\right)^{2}} \leq C_{8}$.

In view of (9), appealing to lemma 1 and Hewitt - Savage zero - one law one gets $\mathrm{P}\left(\mathrm{H}_{\mathrm{k}}\right.$ i.o $)=1$. Hence the proof of the theorem is completed.

\section{Theorem 2}

When $\mathrm{a}_{\mathrm{n}}=[\mathrm{np}], 0<\mathrm{p}<1$, the set of all a.s. limit points of the sequence $\left\{\xi_{\mathrm{n}}, \mathrm{n} \geq 1\right\}$ coincides with $\mathrm{A}_{2}$.

\section{Proof}

Here $\gamma_{\mathrm{n}} \approx \log \log \mathrm{n}$. Hence to prove the assertion it is enough to show that for any $\varepsilon>0, u>0$ and $v>0$,

$$
\mathrm{P}\left(\xi_{\mathrm{n}} \in\left(\mathrm{e}^{\frac{\mathrm{u}-\varepsilon}{\alpha}}, \mathrm{e}^{\frac{\mathrm{u}+\varepsilon}{\alpha}} ; \mathrm{e}^{\frac{\mathrm{v}-\varepsilon}{\alpha}}, \mathrm{e}^{\frac{\mathrm{v}+\varepsilon}{\alpha}}\right) \mathrm{i} . \mathrm{o}\right)=0
$$

whenever $\mathrm{u}+\mathrm{v}>1$ and

$$
\mathrm{P}\left(\xi_{\mathrm{n}} \in\left(\mathrm{e}^{\frac{\mathrm{u}-\varepsilon}{\alpha}}, \mathrm{e}^{\frac{\mathrm{u}+\varepsilon}{\alpha}} ; \mathrm{e}^{\frac{\mathrm{v}-\varepsilon}{\alpha}}, \mathrm{e}^{\frac{\mathrm{v}+\varepsilon}{\alpha}}\right) \text { i.o }\right)=1
$$

whenever $\mathrm{u}+\mathrm{v} \leq 1$.

Let $(u, v)$ be such that $u+v>1$. Define $n_{k}=\left[e^{k}\right]$ and denote the events
$A_{n}=\left\{S_{n} \geq n^{1 / \alpha}(\log n)^{\frac{u-\varepsilon}{\alpha}}, S_{n+a_{n}}-S_{n} \geq p^{1 / \alpha} n^{1 / \alpha}(\log n)^{\frac{v-\varepsilon}{\alpha}}\right\}$

$A_{1, n}=\left\{S_{n} \geq n^{1 / \alpha}(\log n)^{\frac{u-\varepsilon}{\alpha}}\right\}$ and

$A_{2, n}=\left\{S_{n+a_{n}}-S_{n} \geq p^{1 / \alpha} n^{1 / \alpha}(\log n)^{\frac{v-\varepsilon}{\alpha}}\right\}$.

Observe that $A_{n}=\left\{A_{1, n} \cap A_{2, n}\right\}$. To show (19), we appeal to Lemma 2. Since $\left(S_{n}\right)$ and $\left(S_{n+a_{n}}-S_{n}\right)$ are independent, we have

$\mathrm{P}\left(\mathrm{A}_{\mathrm{n}}\right)=\mathrm{P}\left(\mathrm{X}_{1} \geq(\log \mathrm{n})^{\frac{\mathrm{u}-\varepsilon}{\alpha}}\right) \mathrm{P}\left(\mathrm{X}_{2} \geq(\log \mathrm{n})^{\frac{\mathrm{v}-\varepsilon}{\alpha}}\right)$. Choose $\in$ such that $u+v-2 \varepsilon>1$ and using (7), we get for some $C_{1}(>0)$ constant, $\mathrm{P}\left(\mathrm{A}_{\mathrm{n}}\right) \leq \frac{\mathrm{C}_{1}}{(\log \mathrm{n})^{\mathrm{u}+\mathrm{v}-2 \mathrm{a}}}$. Hence $\mathrm{P}\left(\mathrm{A}_{\mathrm{n}}\right) \rightarrow 0$ as $\mathrm{n} \rightarrow \infty$.

We have

$\left(A_{n} \cap A_{n+1}^{c}\right)=A_{n} \cap\left(A_{1, n+1}^{c} \cup A_{2 n+1}\right)=\left(A_{n} \cap A_{1, n+1}^{c}\right) \cup\left(A_{n} \cap A_{2, n+1}^{c}\right)$

Note that $\left(\mathrm{A}_{\mathrm{n}} \cap \mathrm{A}_{1, \mathrm{n}+1}^{\mathrm{c}}\right)=$

$\left\{\begin{array}{l}\mathrm{S}_{\mathrm{n}}>\mathrm{n}^{1 / \alpha}(\log \mathrm{n})^{\frac{\mathrm{u}-\varepsilon}{\alpha}}, \mathrm{S}_{\mathrm{n}+\mathrm{a}_{\mathrm{n}}}-\mathrm{S}_{\mathrm{n}}>\mathrm{p}^{1 / \alpha} \mathrm{n}^{1 / \alpha}(\log \mathrm{n})^{\frac{\mathrm{v}-\varepsilon}{\alpha}} \\ \mathrm{S}_{\mathrm{n}+1}<(\mathrm{n}+1)^{1 / \alpha}\left(\log (\mathrm{n}+1)^{\frac{\mathrm{u}-\varepsilon}{\alpha}}\right.\end{array}\right\}$

$\subseteq\left\{\begin{array}{l}\mathrm{S}_{\mathrm{n}}>\mathrm{n}^{1 / \alpha}(\log \mathrm{n})^{\frac{\mathrm{u}-\varepsilon}{\alpha}}, \mathrm{S}_{\mathrm{n}+\mathrm{a}_{\mathrm{n}}}-\mathrm{S}_{\mathrm{n}}>\mathrm{p}^{1 / \alpha} \mathrm{n}^{1 / \alpha}(\log \mathrm{n})^{\frac{\mathrm{v}-\varepsilon}{\alpha}}, \\ \mathrm{S}_{\mathrm{n}}<(\mathrm{n}+1)^{1 / \alpha}\left(\log (\mathrm{n}+1)^{\frac{\mathrm{u}-\varepsilon}{\alpha}}\right.\end{array}\right\}$

$=\left\{\begin{array}{l}n^{1 / \alpha}(\log n)^{\frac{\mathrm{u}-\varepsilon}{\alpha}}<\mathrm{S}_{\mathrm{n}}<(\mathrm{n}+1)^{1 / \alpha}\left(\log (\mathrm{n}+1)^{\frac{\mathrm{u}-\varepsilon}{\alpha}},\right. \\ \mathrm{S}_{\mathrm{n}+\mathrm{a}_{\mathrm{n}}}-\mathrm{S}_{\mathrm{n}}>\mathrm{p}^{1 / \alpha} \mathrm{n}^{1 / \alpha}(\log \mathrm{n})^{\frac{\mathrm{v}-\varepsilon}{\alpha}}\end{array}\right\}$. Hence

$\mathrm{P}\left(\mathrm{A}_{\mathrm{n}} \cap \mathrm{A}_{1, \mathrm{n}+1}^{\mathrm{c}}\right) \leq \mathrm{P}\left((\log \mathrm{n})^{\frac{\mathrm{u}-\varepsilon}{\alpha}}<\mathrm{X}_{1}<\left(\frac{\mathrm{n}+1}{\mathrm{n}}\right)^{1 / \alpha}\right.$

$\left(\log (\mathrm{n}+1)^{\frac{\mathrm{u}-\varepsilon}{\alpha}}, \mathrm{X}_{2}>\mathrm{p}^{1 / \alpha}(\log \mathrm{n})^{\frac{\mathrm{v}-\varepsilon}{\alpha}}\right)$

$\leq \mathrm{P}\left(\mathrm{u}_{\mathrm{n}}<\mathrm{X}_{1}<\mathrm{v}_{\mathrm{n}}\right) \mathrm{P}\left(\mathrm{X}_{2}>\mathrm{p}^{1 / \alpha}(\log \mathrm{n})^{\frac{\mathrm{v}-\varepsilon}{\alpha}}\right)$

where $u_{n}=(\log n)^{\frac{u-\varepsilon}{\alpha}}$ and $v_{n}=\left(1+\frac{1}{n}\right)^{1 / \alpha}(\log (n+1))^{\frac{u}{\alpha}-\varepsilon}$. Hence we have, 
$P\left(A_{n} \cap A_{1, n+1}^{c}\right) \leq \int_{u_{n}}^{v_{n}} f(x) d x \frac{C_{2}}{(\log n)^{(v-\varepsilon)}}$, where $f$ is the density function of a positive strictly stable r.v, we have density of positive stable law given by $\mathrm{f}(\mathrm{x})=\frac{\mathrm{C}_{3}}{\mathrm{x}^{1+\alpha}}+\frac{\mathrm{C}_{4}}{\mathrm{x}^{1+2 \alpha}}+\mathrm{o}\left(\frac{1}{\mathrm{x}^{1+2 \alpha}}\right)$, where $\mathrm{C}_{3}>0$ and $\mathrm{C}_{4}>0$ are constants. Hence for $\mathrm{x}$ large, one can find $\mathrm{C}>0$ such that $\mathrm{f}(\mathrm{x}) \leq \mathrm{C}\left(\frac{1}{\mathrm{x}^{1+\alpha}}+\frac{1}{\mathrm{x}^{1+2 \alpha}}\right)$. Consequently for $n$ large,

$$
\begin{aligned}
& \mathrm{P}\left(\mathrm{A}_{\mathrm{n}} \cap \mathrm{A}_{1, \mathrm{n}+1}^{\mathrm{c}}\right) \leq \frac{\mathrm{C}}{(\log \mathrm{n})^{\mathrm{v}-\alpha}} \int_{\mathrm{u}_{\mathrm{n}}}^{\mathrm{v}_{\mathrm{n}}}\left(\frac{1}{\mathrm{x}^{1+\alpha}}+\frac{1}{\mathrm{x}^{2+\alpha}}\right) \mathrm{dx} \\
& \leq \frac{\mathrm{C}}{(\log \mathrm{n})^{\mathrm{v}-\alpha}}\left\{\frac{1}{\alpha}\left(\frac{1}{\mathrm{u}_{\mathrm{n}}^{\alpha}}-\frac{1}{\mathrm{v}_{\mathrm{n}}^{\alpha}}\right)+\frac{1}{2 \alpha}\left(\frac{1}{\mathrm{u}_{\mathrm{n}}^{2 \alpha}}-\frac{1}{\mathrm{v}_{\mathrm{n}}^{2 \alpha}}\right)\right\} \\
& =\frac{1}{(\log \mathrm{n})^{\mathrm{u}-\varepsilon}}\left(1-\left(1-\frac{1}{\mathrm{n}}+\frac{\mathrm{C}}{\mathrm{n}^{2}}\right) \frac{(\log \mathrm{n})^{(\mathrm{u}-\varepsilon)}}{\left(\log \mathrm{n}+\log \left(1+\frac{1}{\mathrm{n}}\right)^{(\mathrm{u}-\varepsilon)}\right)}\right) \sim \frac{1}{\mathrm{n}(\log \mathrm{n})^{(\mathrm{u}-\varepsilon)}} \\
& \frac{1}{\mathrm{On}_{\mathrm{n}}^{-\alpha}-\mathrm{v}_{\mathrm{n}}^{-\alpha}=(\log \mathrm{n})^{-(\mathrm{u}-\varepsilon)}-\left(1+\frac{1}{\mathrm{n}}\right)^{-1}\left(\log \mathrm{n}\left(1+\frac{1}{\mathrm{n}}\right)\right)^{-(\mathrm{u}-\varepsilon)}} \\
& \mathrm{u}_{\mathrm{n}}^{2 \alpha}-\frac{1}{\mathrm{v}_{\mathrm{n}}^{2 \alpha}} \leq \frac{\mathrm{C}_{8}}{\mathrm{n}(\log \mathrm{n})^{(\mathrm{u}-\varepsilon)}} \sim \frac{\mathrm{C}}{\mathrm{n}(\log \mathrm{n})^{2 \mathrm{u}-\varepsilon}} \cdot \\
& \text { canilar }
\end{aligned}
$$

For $n$ large say $n \geq N$, from (22) one can show that

$P\left(A_{n} \cap A_{1, n+1}^{c}\right) \leq \frac{C}{n(\log n)^{(u+v-2 a)}}$. Since $u+v-2 \varepsilon>1$, we

have

$$
\sum_{n=1}^{\infty} P\left(A_{n} \cap A_{1, n+1}^{c}\right) \leq C_{9} \sum_{n=1}^{\infty} \frac{1}{n(\log n)^{(u+v-2 a)}}<\infty, \text { for some }
$$$$
\mathrm{C}_{9}>0
$$

Again following similar lines, one can show that $\sum_{n=1}^{\infty} P\left(A_{n} \cap A_{2, n+1}^{c}\right)<\infty$.

Using (25) and (26) in (21), it follows that $\sum_{n=1}^{\infty} P\left(A_{n} \cap A_{n+1}^{c}\right)<\infty$ and hence $P\left(A_{n} \cap A_{n+1}^{c}\right.$ i.o $)=0$, which implies the proof of (21) by Lemma 2 .
Defining $n_{k}=\left[e^{\frac{1}{k^{u+v}}}\right]$ and following the lines of proof similar to those of Theorem 1, the proof of (20) can be obtained and the details are omitted.

\section{Theorem 3}

When $a_{n}=\left[\frac{n}{(\log n)^{q}}\right], q>0$, the set of all a.s. limit points of the sequence $\left\{\xi_{n}, n \geq 1\right\}$ coincides with $A_{2}$.

\section{Proof}

Here $\gamma_{\mathrm{n}} \approx(1+\mathrm{q}) \log \log \mathrm{n}$ and from lemmas 4 and 5, we know that

$\operatorname{Lim} \operatorname{Inf}_{n \rightarrow \infty}(\operatorname{Sup})\left(\frac{T_{n}}{a_{n}^{1 / \alpha}}\right)^{\frac{1}{(1+q) \log \log n}}=1\left(e^{1 / \alpha}\right)$ a.s .

Hence $\xi_{n}=\left\{\left(\frac{S_{n}}{n^{1 / \alpha}}\right)^{\theta_{n}},\left(\frac{T_{n}}{a_{n}^{1 / \alpha}}\right)^{\frac{\theta_{n}}{(1+q)}}\right\}$.

Proceeding on the lines of Theorem 2, the a.s. limit set can be shown to be $A_{2}$. The details are omitted.

\section{ACKNOWLEDGEMENTS}

The authors express their sincere thanks to the two referees for their positive responses and valuable comments in this work. They would also like to express their gratitude to the publishers for their invitation to contribute an article. "Research supported by UGC Major Research Project F. No: $34-156$ / 2008 (SR).

\section{REFERENCES}

[1] R. P. Pakshirajan, and R. Vasudeva, "A LIL for stable summands", Trans. Am. Math. Soc., vol. 232, pp. 33-42, 1977.

[2] J. Chover, "A law of the iterated logarithm for stable summands", Proc. Am. Math., vol. 17, pp. 441-443, 1966.

[3] R. Vasudeva, and G. Divanji, "Almost sure limit set of the vector sequence of partial sums and delayed sums", CSA Bulletin, vol. 39, Nos. 155-156, pp. 151-161, 1990.

[4] F. Spitzer, Principles of Random Walk. Van Nostrand: Princeton, New Jercey, 1964.

[5] O. B. Nielsen, "On the rate of growth of the partial maxima of sequence of independent identically distributed random variables" Math. Scand., vol. 9, pp. 383-394, 1961.

[6] W. Feller, An Introduction to Probability Theory and its Applications" Fourth Wiley Eastern reprint, Wiley Eastern Limited: India, 1986, Vol. II.

[7] R. Vasudeva, and G. Divanji, "LIL for delayed sums under a nonidentically distributed setup" TPRBAU, vol. 37, No. 3, pp. 534$542,1992$. 